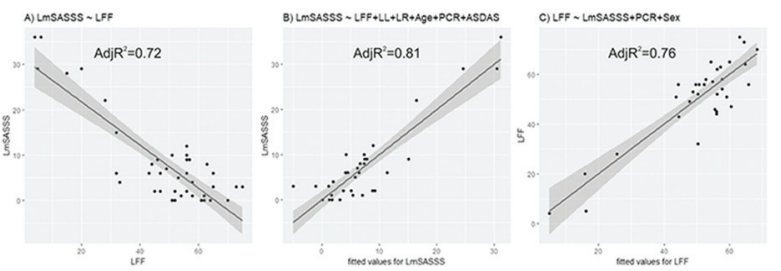

and during pregnancy; moreover, in 4 cases AS was interpreted as contraindication to pregnancy.

$107(35.4 \%)$ respondents believe that AS medications should be discontinued during pregnancy planning and conception, 75 (24.8\%) subjects accepted possible use of AS therapy during this period, while 120 $(39.8 \%)$ didn't know the answer. $15.2 \%$ of respondents were ready to continue on NSAIDs, 20 (6.6\%) - on glucocorticoids and sulfasalazine, and $21(7 \%)$ - on biological drugs (GEBD). Of those who are ready to continue on AS medications at conception, 46 women $(61.3 \%)$ consulted pregnancy planning with a rheumatologist. Respondents with higher education were slightly more likely to continue on AS therapy at conception (29\%) compared to women with vocational college education (11.8\%). Age, AS duration, or marital status had no influence on patients' attitude to AS therapy during pregnancy planning and conception.

Conclusion: The majority of surveyed female patients changed their attitude to pregnancy after establishing AS diagnosis, although only $13 \%$ of them were ready to avoid pregnancy due to AS. No more than $50 \%$ of respondents were consulted a rheumatologist before planning pregnancy, and up to one third of them were not receive all expected answers to their questions and recommendations. Only $1 / 4$ of participants accept the idea of continuing AS therapy during pregnancy planning and conception. Disclosure of Interests: None declared

DOI: 10.1136/annrheumdis-2019-eular.3958

\section{SAT0327 SEGMENTAL RELATIONSHIP BETWEEN MOBILITY, STRUCTURAL DAMAGE AND DISEASE ACTIVITY IN AXIAL SPONDYLOARTHRITIS}

Juan L. Garrido-Castro ${ }^{1}$, Inmaculada Concepcion Aranda-Valera ${ }^{1}$,

Cristina Gonzalez-Navas ${ }^{1}$, Pedro Machado ${ }^{2}$, Philip Gardiner ${ }^{3}$, Joan Condell ${ }^{3}$, Eduardo Collantes Estevez ${ }^{1} .{ }^{1} I M I B I C$, Cordoba, Spain; ${ }^{2}$ UCL, London, United Kingdom; ${ }^{3}$ WHSCT, Londonderry, United Kingdom

Background: Axial Spondyloarthritis (axSpA) is characterised by progressive loss of spinal mobility, due to inflammation and structural damage. Conventional mobility tests have been used alongside radiographic structural damage and disease activity scores in order to explore the overall relationship between spinal mobility, structural damage and disease activity. This has rarely been done at the segmental level e.g. at the level of the lumbar spine only. The ViMove inertial motion sensor-based system allows segmental spinal mobility to be directly measured, namely at the lumbar spine level.

Objectives: To analyse the relationship between mobility, structural damage and disease activity in the lumbar spine of patients with axSpA.

Methods: Lumbar spinal mobility was measured using the the ViMove system with a pair of sensors located at L1 and the sacrum. Lumbar ROM in the three planes (anterior flexion - LFF, extension - LFE, lateral flexion - LL, and rotation - LR) was measured. Radiographs were obtained to calculate the lumbar part of the mSASSS (LmSASSS). Other outcome measures were: BASMI, Schober and lateral flexion (measured with a tape), BASDAI and ASDAS-CRP. Subgroups of patients with high and low levels of lumbar structural damage were defined, by dichotomising the population based on the median LmSASSS (5.5). Pearson correlations between measures, Student $T$ tests for significant differences between groups and linear regression analysis, to analyse relationship between structural damage and mobility, were obtained.

Results: 44 axSpA patients were recruited from the COSPAR cohort of the Reina Sofia University Hospital (39\% females, age $43 \pm 12$ years, disease duration $17 \pm 14$ years, BMI 26 \pm 3 ). All mobility measurements (except for LFE) in the severe damage group were significantly reduced compared to the group with less damage $(p<0.001)$. BASDAI and ASDAS were not significantly different between these groups. LmSASSS correlated $(p<0.001)$ with all mobility measures obtained by IMU system and conventional metrology, especially with LFF, but not with activity indexes Only a weak relation between BASDAI and LFE was found $(p<0.05)$. A linear regression for analysing relationship between LmSASSS and LFF including as variables: age, sex, evolution time, BMI, PCR, ASDAS, LFF, LFE, LL, LR and LmSASSS was done. LmSASSS linear regression using only LFF obtained an adjusted $\mathrm{R}^{2}$ of 0.72 (Figure-A). Introducing the rest of variables, the most significant for LmSASSS was LFF $(p<0.001)$ and the other mobility measures (LL, LR with $\mathrm{p}<0.05$ ) and age, PCR and ASDAS $(p<0.01)$ with an adjusted $R 2$ of 0.81 (Figure-B). For LFF, LmSASSS was the most significant $(p<0.001)$, with Sex $(p<0.01)$ and PCR $(p<0.05)$ included, obtaining an adjusted R2 of 0.76 (Figure-C). Differences between models and fitted values (RMSE) were 3.8 units for LmSASSS and $7.7^{\circ}$ for LFF.

Conclusion: Other studies have shown a relationship between mobility structural damage and disease activity using a more global assessment Our study focuses on the analysis of structural damage and mobility in the lumbar vertebrae, and our results suggest that structural damage is the dominant factor determining spinal mobility in this region (especially lumbar frontal flexion). Further studies are needed combining these results with MRI.

Acknowledgement: This study was funded by FOREUM (www.foreum. org), Junta de Andalucia (PIN-0079-2016) and XXI Research Promotion Program of the University of Córdoba (Spain).

Disclosure of Interests: Juan L. Garrido-Castro: None declared, Inmaculada Concepcion Aranda-Valera: None declared, Cristina Gonzalez-Navas: None declared, Pedro Machado Consultant for: Abbvie, BMS, Celgene Janssen, MSD, Novartis, Pfizer, Roche and UCB, Speakers bureau: Abbvie, BMS, Celgene, Janssen, MSD, Novartis, Pfizer, Roche and UCB, Philip Gardiner: None declared, Joan Condell: None declared, Eduardo Collantes Estevez: None declared

DOI: 10.1136/annrheumdis-2019-eular.1035

\section{SAT0328 PATIENT-REPORTED ATTITUDES TOWARDS AXIAL SPONDYLOARTHRITIS: RESULTS FROM THE EMAS SURVEY}

Marco Garrido-Cumbrera ${ }^{1,2}$, Laure Gossec ${ }^{3,4}$, Denis Poddubnyy ${ }^{5,6}$, Souzi Makri ${ }^{7}$ Victoria Navarro-Compán ${ }^{8}$, Christine Bundy ${ }^{9}$, Carlos Delgado Domínguez ${ }^{1}$, Olta Braçe 1 , Sergio Sanz-Gómez ${ }^{1}$, Raj Mahapatra ${ }^{10}$. ${ }^{1}$ Universidad de Sevilla, Health and Territory Research (HTR), Seville, Spain; ${ }^{2}$ CEADE, Madrid, Spain; ${ }^{3}$ Sorbonne Université, Institut Pierre Louis d'Epidémiologie et de Santé Publique (iPLESP), Paris, France; ${ }^{4}$ Pitié Salpêtrière Hospital, AP-HP, Paris, France; ${ }^{5}$ Charité-Universitätsmedizin Berlin, Berlin, Germany; ${ }^{6}$ German Rheumatism Research Centre, Berlin, Germany; ${ }^{7}$ Cyprus League Against Rheumatism, Nicosia, Cyprus; ${ }^{8}$ Hospital Universitario La Paz, IdiPaz, Madrid, Spain; ${ }^{9}$ Cardiff University, Cardiff, United Kingdom; ${ }^{10}$ Ankylosing Spondylitis International Federation (ASIF), London, United Kingdom

Background: Understanding patient disease-related attitudes plays an important role in effective management of axial spondyloarthritis (axSpA). Nevertheless, the patient perspective remains insufficiently explored.

Objectives: To describe patients' axSpA-related fears and hopes and their associations with sociodemographic, disease outcomes and psychological distress.

Methods: The European Map of Axial Spondyloarthritis (EMAS), conducted from July 2017 to February 2018, was a cross-sectional on-line survey of unselected patients with self-reported axSpA from Austria, Belgium, France, Germany, Italy, Netherlands, Norway, Russia, Slovenia, Spain, Sweden, Switzerland, and the UK. Participants were recruited through an online panel and patient organizations. Participant's axSpArelated fears and hopes were freely expressed through open-ended ques tions (except in France where multiple-choice items were used). Thematic analysis, using the French categories and data-driven codes, was performed and frequencies of specific fears and hopes were subsequently calculated. Associations between disease-related fears and hopes with sociodemographic characteristics, disease outcomes (BASDAI, self-reported spinal stiffness and functional limitation), and psychological distress (General Health Questionnaire, GHQ-12; with a cut-off point of $>3$ ) were

\begin{tabular}{|c|c|c|c|c|c|c|c|c|c|}
\hline & $N$ & Flexion & Extension & Lateral & Rotation & LmSASSS & BASMI & ASDAS & BASDAI \\
\hline Total & 44 & $48.8(17.4)$ & $10.5(13.4)$ & $42.7(17.0)$ & $24.8(8.8)$ & $8.0(9.7)$ & $3.0(1.9)$ & $1.9(1.1)$ & $3.7(2.0)$ \\
\hline Low & 22 & $57.2(10.0)$ & $12.7(16.9)$ & $49.4(10.1)$ & $27.9(7.6)$ & $1.8(1.5)$ & $2.3(1.4)$ & $1.8(1.2)$ & $3.6(1.9)$ \\
\hline High & 22 & $39.6(19.7)$ & $7.7(9.1)$ & $35.1(20.0)$ & $21.1(8.4)$ & $14.2(10.5)$ & $3.9(2.1)$ & $2.2(1.0)$ & $3.7(2.1)$ \\
\hline
\end{tabular}

